\title{
Intraperitoneal Chemotherapy
}

National Cancer Institute

\section{Source}

National Cancer Institute. Intraperitoneal Chemotherapy. NCI Thesaurus. Code C15581.

The administration of pharmaceutical agents directly into the abdominal or peritoneal cavity. This technique is widely utilized for the chemotherapeutic treatment of advanced ovarian cancer. 